\title{
AVALIAÇÃO DO NIVEL DE ESTRESSE EM ENFERMEIROS DA EMERGÊNCIA DE UM HOSPITAL DE GRANDE PORTE
}

\section{EVALUATION OF THE LEVEL OF STRESS IN EMERGENCY NURSES OF A LARGE HOSPITAL}

Recebido em: 28/08/2018.

Aceito em: 27/11/2018.

\author{
Cizélia Barreto Santos ${ }^{1}$ \\ Mona Freitas Santos ${ }^{2}$ \\ Kay Santos Amparo 3 \\ Samuel Santos Souza ${ }^{4}$ \\ Renara Meira Gomes \\ Mirian Souza Pereira da Silva ${ }^{6}$
}

\section{RESUMO}

Objetivo: avaliar o nível de estresse dos enfermeiros que atuam na emergência de um hospital de grande pobrte no interior do estado da Bahia. Método: estudo epidemiológico transversal de caráter descritivo, aplicado em 27 enfermeiros que atuam na Unidade de Urgência/emergência de um hospital público localizado no nordeste brasileiro. Para coleta dos dados, utilizou-se a Escala Bianchi de Stress (EBS), onde foi calculada a média dos itens que compõem cada domínio. Os dados foram tabulados e analisados utilizando o programa SPSS versão 21. A variável de desfecho foi o nível de estresse, categorizado em: abaixo da média (até 3,7) e acima da média (a partir de 3,8). As variáveis independentes foram as características sociodemográficas e ocupacionais. Resultados: dos 27 enfermeiros 29,6\% representando baixo nível de estresse, 70,4\% com médio nível de estresse, dos enfermeiros que estão acima da média de estresse

1 Graduanda em Enfermagem pela Faculdade de Tecnologia e Ciências (FTC).

E-mail: cizelia_barreto@hotmail.com

2 Especialista em Saúde Coletiva pela Universidade Estadual do Sudoeste da Bahia (UESB). Docente da Faculdade de Tecnologia e Ciêncas (FTC).

E-mail: monafreitassantos@gmail.com

3 Mestranda em Enfermagem e Saúde pela Universidade Estadual do Sudoeste da Bahia (UESB). Docente da Faculdade de Tecnologia e Ciêncas (FTC).

E-mail: kayamparo@hotmail.com

4 Especialista em saúde Coletiva pela Universidade Estadual do Sudoeste da Bahia (UESB). E-mail: samuelsantossouza@hotmail.com

5 Graduanda em Enfermagem pela Faculdade de Tecnologia e Ciências (FTC).

E-mail: nara_rhema@hotmail.com

6 Graduanda em Enfermagem pela Faculdade de Tecnologia e Ciências (FTC).

E-mail:mi.sps@hotmail.com 
91,7\% são mulheres, $58,3 \%$ têm idade entre 30 a 39 anos, $80 \%$ católicos, $75,0 \%$ de cor parda, $83,3 \%$ casados e $91,7 \%$ têm filhos, $83,3 \%$ possuem especialização, $41,7 \%$ têm de 11 a 20 anos de formado, $66 \%$ atuam de 1 a 6 anos na unidade $58,3 \%$ trabalham em revezamento, que 63,6 são estatutário, 66,7\% possuem outro vínculo, 83,3\% são insatisfeitos com a renda. Conclusão: enfermeiros atuantes em unidade de urgência e emergência estão sujeitos ao desenvolvimento do estresse, faz-se necessário um olhar diferenciado a esse profissional, pois é preciso está bem de saúde para cuidar da saúde de outros.

Palavras-chave: Emergência. Enfermeiro. Estresse ocupacional.

\section{ABSTRACT}

Objective: to evaluate the stress level of nurses who work in the emergence of a large hospital in the state of Bahia. Method: cross - sectional epidemiological study of descriptive character, applied in 27 nurses who work in the Emergency Unit of a public hospital located in the Brazilian Northeast. For data collection, the Bianchi Stress Scale (EBS) was used to calculate the average of the items that make up each domain. Data were tabulated and analyzed using the SPSS software version 21. The outcome variable was the stress level, categorized as below average (up to 3.7) and above average (from 3.8). The independent variables were sociodemographic and occupational characteristics. Results: Of the 27 nurses, $29.6 \%$ represented a low level of stress, $70.4 \%$ had a medium level of stress, nurses who were above the mean of stress, $91.7 \%$ were women, $58.3 \%$ were between 30 and $39.7 \%$ have children, $83.3 \%$ have specialization, $41.7 \%$ have 11 to 20 years of education, $66 \%$ of them are married, they work from 1 to 6 years in the unit $58.3 \%$ work in relay, 63.6 are statutory, $66.7 \%$ have another link, $83.3 \%$ are dissatisfied with income. Conclusion: nurses working in emergency and emergency units are subject to the development of stress, it is necessary to have a different look at this professional, since it is necessary to be in good health to take care of the health of others.

Keywords: Emergencies. Nurse. Occupational stress.

\section{INTRODUÇÃO}

A enfermagem em urgência e emergência tem avançado de maneira significativa nos últimos anos por ser uma especialidade de grande relevância e pelo elevado número de acidentes e de violência urbana que culminam, na maioria das vezes, com pessoas em estado crítico e com risco de morte. Nesta perspectiva compreendemos que a instituição 
hospitalar é fator desencadeante para a existência de estresse no enfermeiro que atua no setor de urgência e emergência (AVELINO et al., 2013).

É notório afirmar que atuar em situações emergenciais, constitui-se uma atividade desgastante pelo quadro clínico, muitas vezes grave, em que o paciente apresenta; assimilando isso a impaciência dos familiares e o quantitativo de profissionais existente para o desenvolvimento das atividades (KIRCHHOF et al., 2016).

Diante desse cenário, pode-se constatar que o estresse é uma das principais causas de adoecimentos dos profissionais enfermeiros, influenciado pelos determinantes e condicionantes vivenciados no local de trabalho (AVELINO et al., 2013). Corrobora nessa mesma perspectiva Bezerra, Silva e Ramos, (2012), ao constatarem que as fontes geradoras de estresse relacionam-se principalmente com a forma de organização do processo de trabalho, como também, as relações interpessoais que nele são estabelecidas.

Apesar do estresse ainda não caracterizar uma doença do profissional enfermeiro, estes são afetados diretamente, constituindo-se agravo potencial de grande intensidade. Tal problemática merece uma atenção especial, visto que pode desencadear sérios problemas na saúde dos enfermeiros atuantes em unidades de urgência e emergência (OLIVEIRA et al., 2013).

Em suma, sabe-se que os fatores responsáveis pelo adoecimento do profissional são variados, porém, cada um responde de forma diferenciada diante de um agente estressor (FERREIRA, 2016).

Considerando que a emergência de um hospital é a porta de entrada e um ambiente onde requer autocontrole e agilidade da equipe que está à frente desse trabalho; como a maior equipe de um hospital é a de enfermagem, na emergência não seria diferente, por isso esses profissionais estão mais propensos a desenvolverem o desgaste físico e mental pelo acumulo de tarefas e conflitos com a equipe.

Para tanto, este estudo tem por objetivo avaliar o nível de estresse dos enfermeiros que atuam na emergência de um hospital de grande porte no interior do estado da Bahia.

\section{MÉTODOS}

Trata se de um estudo epidemiológico transversal de caráter descritivo, aplicado em 27 enfermeiros que atuam na Unidade de Urgência e Emergência.

A Unidade de Urgência e Emergência do referido hospital público localizado no nordeste brasileiro, é gerenciado pelo estado, e serve como unidade referência para 26 municípios. Trata-se de um serviço de alta complexidade, que possui uma grande demanda diária de pacientes nas diversas especialidades médicas, atende em regime de plantão permanente, conta com 175 leitos para o Cadastro Nacional de Estabelecimentos de Saúde - CNES (contudo a um quantitativo de leitos maior devido aos extras existentes), sendo 24 leitos destinados à unidade de emergência, os quais são utilizados 
também para internação de longa permanência pela falta de leitos para transferência inter-hospitalar. A unidade de emergência conta com 28 enfermeiros assistencialistas.

Os sujeitos da pesquisa constituíram-se de 27 enfermeiros que atuam na Unidade de Urgência e Emergência. Os critérios de inclusão foram: ser profissionais enfermeiros (as) de ambos os sexos que atuavam na emergência deste hospital a mais seis meses, na assistência ou coordenação, e que após os esclarecimentos da pesquisa assinasse o Termo de Consentimento Livre e Esclarecido -TCLE. Como critério de exclusão optou-se por não incluir os profissionais enfermeiros (as) do setor que estavam em férias ou afastados por algum motivo (atestados, licenças, etc.), bem como, aqueles que recusaram a assinar o TCLE.

Para coleta dos dados utilizou-se a Escala Bianchi de Stress (EBS), prevista para avaliar o nível de estresse do enfermeiro hospitalar. O instrumento foi constituído por um questionário contendo variáveis sociodemográficas, ocupacionais e estressores na atuação do enfermeiro, com 51 itens usando a escala tipo Likert, com variação de 1 a 7 .

Para verificar o nível de estresse de cada enfermeiro foi calculada a média dos itens que compõem cada domínio, excluindo-se o número de zeros marcados e os valores omissos. Foi considerado nível de estresse com a seguinte pontuação: igual ou abaixo de 3,0, nível de estresse baixo; entre 3,1 e 5,9, nível de estresse médio; e igual ou acima de 6,0, nível de estresse alto. Os dados foram tabulados e analisados utilizando o programa SPSS versão 21.

E para analisar as características sociodemográficas e ocupacionais segundo a média de estresse, a variável de desfecho foi o nível de estresse de enfermeiros, categorizado em: abaixo da média (até 3,7) e acima da média (acima de 3,7). Essa dicotomização foi estratificada a partir da média do grupo na EBS.

As variáveis independentes foram estudas por grupos. Características sociodemográficas: sexo (masculino e feminino); idade em anos e dividida em três faixas etárias (20 a 29, 30 a 39 e 40 ou mais); religião (católica, evangélica e outras); raça/ cor (branca, parda e preta); estado civil (solteiro, casado, divorciado) e filhos (sim e não). Características ocupacionais: pós-graduação (especialização e mestrado), tempo de formado em anos e dividida em três faixas etárias (1 a 10, 11 a 20 e 21 ou mais); turno (dia, noite e revezamento); outro vínculo (sim e não); tipo de vínculo (estatutário e celetista); tempo na unidade em anos e dividida em duas faixas etárias ( 1 a 5 , e 6 ou mais) e satisfeito com a renda (sim e não).

Para avaliar a confiabilidade interna da escala utilizou-se o coeficiente Alfa de Cronbach e obteve na escala total $=0,93$, considerando os valores acima de 0,7 como satisfatórios (MAROCO; GARCIA-MARQUES, 2013).

A pesquisa cumpriu os preceitos éticos e legais exigidos, sendo aprovada pelo Comitê de Ética em Pesquisa (CEP) do Instituto Mantenedor de Ensino Superior da Bahia (IMES) com CAAE n ${ }^{\circ}$ 82949018.0.0000.5032 e todos os participantes assinaram o Termo de Consentimento Livre e Esclarecido. 


\section{RESULTADOS}

A amostra do estudo foi constituída por 27 enfermeiros assistencialistas, atuantes na unidade de urgência e emergência do hospital, distribuídos em três setores anexos (trauma, estabilização e emergência pediátrica).

A Tabela 1 apresenta o nível individual de classificação de estresse dos enfermeiros. Segundo a EBS obteve-se um total de 29,6\% de enfermeiros representando baixo nível de estresse, 70,4\% com médio nível de estresse, e nenhum com alto nível de estresse $0,0 \%$.

Tabela 1 - Distribuição do nível de estresse de enfermeiros $(n=27)$ atuantes na emergência de um hospital do interior da Bahia. Jequié, Bahia, Brasil, 2018.

\begin{tabular}{|c|c|c|c|}
\hline \multirow{3}{*}{ Média EBS } & \multicolumn{3}{|c|}{ Classificação do nível de estresse } \\
\cline { 2 - 4 } & Baixo (até 03 pontos) & Médio $(3,1-5,9$ pontos $)$ & Alto $(\geq 06$ pontos) \\
\cline { 2 - 4 } & $\mathrm{n}(\%)$ & $\mathrm{n}(\%)$ & $\mathrm{n}(\%)$ \\
\hline 3,7 & $8(29,6)$ & $19(70,4)$ & $0(0,0)$ \\
\hline
\end{tabular}

A Tabela 2 apresenta os dados das características sociodemográficas segundo a média de estresse. Demonstrou que 91,7\% dos enfermeiros que estão acima da média de estresse são do sexo feminino.

Quanto à faixa etária, os enfermeiros que estão acima da média de estresse, têm idade variada entre 30 a 39 anos, representado 58,3\%, pode ser observado ainda na Tabela 2 que dos enfermeiros que estão acima da média do nível de estresse, $80 \%$ declararam ser católicos, 75,0\% de cor parda, 83,3\% são casados e 91,7\% referiram ter filhos.

A Tabela 3 refere-se às características ocupacionais segundo a média de estresse, indica que dos que estão acima da média de estresse, 83,3\% possuem grau de instrução á nível de especialização. Em relação ao tempo de formação 41,7\% dos profissionais acima da média de estresse, têm de 11 a 20 anos de formado, e $66 \%$ dos que indicaram nível de estresse acima da média, têm de 1 a 6 anos de atuação na unidade.

A maioria dos enfermeiros que estão acima do nível de estresse, 58,3\% trabalham em uma escala de serviço do tipo revezamento dia/noite. No tocante ao tipo de vínculo empregatício, os resultados demonstram que 63,6\% dos enfermeiros acima da média são estatutário, e 66,7\% possuem outro vínculo empregatício respectivamente. Por fim, em relação à satisfação com a renda, 83,3\% declararam não estarem satisfeitos. 
Tabela 2 - Características sociodemográficas segundo nível de estresse. Jequié, Bahia, Brasil, 2018.

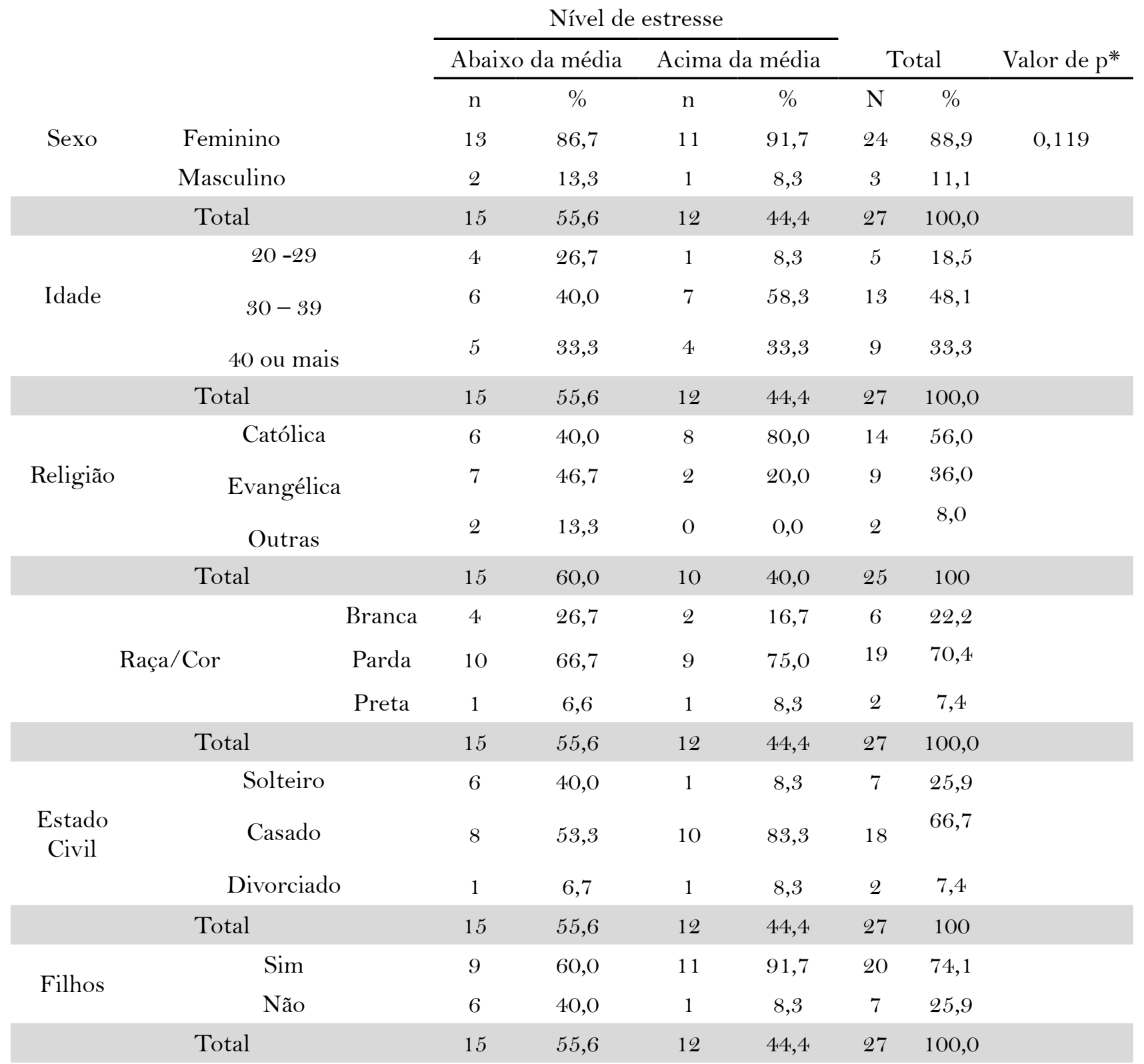

*Teste Qui-uadrado 
Tabela 3 - Características ocupacionais segundo nível de estresse. Jequié, Bahia, Brasil, 2018.

\begin{tabular}{|c|c|c|c|c|c|c|c|c|}
\hline & & \multicolumn{4}{|c|}{ Média de estresse } & & & \multirow[b]{2}{*}{ Valor de $\mathrm{p}^{*}$} \\
\hline & & \multicolumn{2}{|c|}{ Abaixo da media } & \multicolumn{2}{|c|}{ Acima da média } & \multicolumn{2}{|c|}{ Total } & \\
\hline \multirow{3}{*}{$\begin{array}{l}\text { Pós-gra- } \\
\text { duação }\end{array}$} & & $\mathrm{n}$ & $\%$ & $\mathrm{~N}$ & $\%$ & $\mathrm{n}$ & $\%$ & \\
\hline & Especialização & 13 & 100,0 & 10 & 83,3 & 23 & 92,0 & 0,125 \\
\hline & Mestrado & $\mathrm{O}$ & $\mathrm{O}, \mathrm{O}$ & 2 & 16,7 & 2 & 8,0 & \\
\hline \multicolumn{2}{|r|}{ Total } & 13 & 52,0 & 12 & 48,0 & 25 & 100,0 & \\
\hline \multirow{4}{*}{$\begin{array}{l}\text { Tempo de } \\
\text { formado }\end{array}$} & $1-10$ & 9 & 60,0 & 4 & 33,3 & 13 & 48,2 & \multirow{3}{*}{0,382} \\
\hline & $11-20$ & 4 & 26,7 & 5 & 41,7 & 9 & 33,3 & \\
\hline & 21 ou mais & 2 & 13,3 & 3 & 25,0 & 5 & 18,5 & \\
\hline & Total & 15 & 55,6 & 12 & 44,4 & 27 & 100,0 & \\
\hline \multirow{3}{*}{ Turno } & Diurno & 6 & 40,0 & 3 & 25,0 & 9 & 33,3 & \multirow{3}{*}{0,714} \\
\hline & Noturno & 2 & 13,3 & 2 & 16,7 & 4 & 14,8 & \\
\hline & Revezamento & 7 & 47,7 & 7 & 58,3 & 14 & 51,9 & \\
\hline \multicolumn{2}{|c|}{ Total } & 15 & 55,6 & 12 & 44,4 & 27 & 100,0 & \multirow{3}{*}{0,168} \\
\hline \multirow{2}{*}{$\begin{array}{l}\text { Outro vín- } \\
\text { culo }\end{array}$} & Sim & 6 & 40,0 & 8 & 66,7 & 14 & 51,9 & \\
\hline & Não & 9 & 60,0 & 4 & 33,3 & 13 & 48,1 & \\
\hline \multicolumn{2}{|r|}{ Total } & 15 & 55,6 & 12 & 44,4 & 27 & 100,0 & \multirow{3}{*}{0,495} \\
\hline \multirow{2}{*}{$\begin{array}{l}\text { Tipo de vín- } \\
\text { culo }\end{array}$} & Estatutário & 7 & 50,0 & 7 & 63,6 & 14 & 56,0 & \\
\hline & Celetista & 7 & 50,0 & 4 & 36,4 & 11 & 44,0 & \\
\hline \multicolumn{2}{|c|}{ Total } & 14 & 56,0 & 11 & 44 & 25 & 100,0 & \multirow{3}{*}{0,722} \\
\hline \multirow{3}{*}{$\begin{array}{l}\text { Tempo na } \\
\text { unidade }\end{array}$} & $1-6$ & 9 & 60,0 & 8 & 66,7 & 17 & 63,0 & \\
\hline & 6 ou mais & 6 & 40,0 & 4 & 33,3 & 10 & 37,0 & \\
\hline & Total & 15 & 55,6 & 12 & 44,4 & 27 & 100,0 & \multirow{3}{*}{0,535} \\
\hline \multirow{2}{*}{$\begin{array}{l}\text { Satisfação } \\
\text { com a renda }\end{array}$} & Sim & 4 & 26,7 & 2 & 16,7 & 6 & 22,2 & \\
\hline & Não & 11 & 73,3 & 10 & 83,3 & 21 & 77,8 & \\
\hline \multicolumn{2}{|r|}{ Total } & 15 & 55,6 & 12 & 44,4 & 27 & 100,0 & \\
\hline
\end{tabular}

*Teste Qui-quadrado

De acordo com os dados da Tabela 2 e 3, nenhuma variável se associou significativamente $(\mathrm{p}<0,05)$ a média de estresse. Entretanto, alguns comentários acerca do escore médio da EBS obtido em cada quesito serão realizados.

\section{DISCUSSÃO}

Apesar do estresse ainda não caracterizar uma doença dos profissionais enfermeiros, estes são afetados diretamente, constituindo-se um agravo potencial de grande intensidade (OLIVEIRA et al., 2013). 
Após a aplicação da EBS foi possível perceber que o estresse é um fator presente no cotidiano laboral dos enfermeiros que atuam na unidade de urgência e emergência do hospital. Os dados expressos revelaram que 70,4\% dos enfermeiros entrevistados apontam para médio nível de estresse, representando um quantitativo elevado.

Um estudo realizado utilizando escala semelhante à EBS demonstra o mesmo resultado em relação à classificação do nível de estresse por meio da avaliação dos escores dos profissionais (AVELINO et al., 2013). Nesse sentido, faz-se necessário uma investigação acerca das causas pelas quais se evidenciou médio nível de estresse na população investigada.

Em relação às características sociodemográficas, de acordo com os dados que foram tabelados o grande percentil é composto por mulheres, que representaram 88,9\% da amostra total, e 91,7\% que expressaram estar acima da média de estresse também são do sexo feminino. Corrobora de igual maneira Avelino et al. (2013), ao caracterizar um grupo estudado, observando o predomínio do sexo feminino, assim como Araújo et al. (2017), demonstrando que mais da metade do percentual era constituído por mulheres, fator característico dessa profissão. É necessário ressaltar que as mulheres têm dupla jornada de trabalho, o que as tornam vulneráveis a desencadear o estresse.

No que tange a faixa etária entre 30 e 39 anos os dados apontaram que 58,3\% dos enfermeiros apresentaram média de estresse acima do nível médio ressaltando o fato de estarem em seu período ativo e produtivo (OLIVEIRA et al., 2013). Porém, os aspectos físicos e mentais têm sido apresentados na literatura como responsáveis para o surgimento de diversos fatores que desencadeiam o estresse, independentes da idade (PETERSEN; MARZIALE, 2017).

Quanto ao estado civil e presença de filhos, observa-se que há um número relativo expressivo acima da média de estresse de enfermeiros que são casados e que possuem filhos. Evidentemente, ser casado e possuir filhos serviram de agravante para o estresse, considerando que as preocupações do indivíduo aumentam, principalmente em relação às responsabilidades inerentes à família (TRETTENE et al., 2016).

Alguns componentes do trabalho demonstram-se como estressores e podem contribuir para o desenvolvimento do estresse ocupacional, como revela a Tabela 3. Em relação ao grau de instrução predominaram os sujeitos com curso de especialização acima da média de estresse, demais estudos apontam esse mesmo percentual (AVELINO et al., 2013; TRETTENE et al., 2016).

Os resultados mencionaram que o turno tipo revezamento, que é o trabalho em períodos de turno diurno e noturno demonstraram elevado nível de estresse, apontado por $58,3 \%$ dos enfermeiros que estão acima da média, corroborando com estudo exploratório, cujo objetivo foi determinar o nível de estresse de enfermeiros, realizado no município de Vitória - ES (BATISTA; BIANCHI, 2013). Possível hipótese para esse achado pode estar associado, ao fato de que esquema de revezamento de turnos de trabalho influência na percepção do estresse, interferindo na vida do profissional no âmbito familiar (LIMA; BIANCHI, 2010). 
O presente estudo aponta que os enfermeiros com vínculo empregatício estatutários apresentaram maiores níveis de estresse, contudo, Petersen e Marziale (2017), observaram que uma proporção importante de trabalhadores celetistas e/ ou terceirizados estão mais predispostos a apresentarem níveis elevados de estresse, devido as preocupações e inseguranças em relação ao vínculo. Há evidências em outros estudos de que a precarização do vínculo empregatício implica em constrangimento e hipossuficiência a este conjunto de trabalhadores. Considerando ainda que o vínculo precário aponta uma política discriminatória e excludente, que se efetiva na contramão a luta histórica da categoria de enfermagem pelo fortalecimento do sistema público de saúde (RIBEIRO; SOUZA; SILVA, 2014).

Notou-se que possuir outro vínculo propicia em aumento do estresse. Haja vista que múltiplos vínculos empregatícios, com todas as responsabilidades inerentes, expõem a maior probabilidade para o desenvolvimento de tal problemática (TRETTENE et al., 2016).

No que se refere à remuneração é notória a insatisfação, que repercute de modo atenuante pelo coletivo, o que pode acarretar potenciais prejuízos psicológicos, visto que $83,3 \%$ de enfermeiros com nível elevado de estresse mencionam não estar satisfeitos com a sua renda (WISNIEWSKI et al., 2015).

\section{CONCLUSÃO}

Este estudo demonstrou que os enfermeiros atuantes em unidade de urgência e emergência estão sujeitos ao desenvolvimento do estresse, decorrente a diversos fatores existentes, como idade, estado civil, tipo e quantidade de vínculo empregatício e ter filhos, assim como fatores ocupacionais, relacionados ao trabalho.

Sabe-se que o estresse pode desencadear distúrbios e doenças, prejudicando a saúde do indivíduo afetado, repercutindo na assistência prestada. Diante de tal problemática faz-se necessário um olhar diferenciado a esse profissional, pois é preciso está bem de saúde para cuidar da saúde de outros.

Ao identificar a média de estresse dos enfermeiros atuantes no serviço de urgência/emergência dessa unidade hospitalar, o estudo buscou contribuir com informações que possam subsidiar a adoção de medidas que adequem as características e o perfil dos profissionais com a demanda e rotina da unidade.

\section{REFERÊNCIAS}

ARAUJO, M. A. N. de et al. Perfil sociodemográfico dos enfermeiros da rede hospitalar. Rev. enferm. UFPE on line, v. 11, n. supl.11, p. 4716-4725, nov. 2017.

AVELINO, F. V. S. D. et al. Estresse em enfermeiros do setor de urgência e emergência. Revista de Enfermagem da UFPI, v. 2, n. 3, p. 4-10, 9 set. 2013. 
BATISTA, K. de M.; BIANCHI, E. R. F. La relación estrés, resistencia y turno de trabajo en enfermeros de un hospital de enseñanza. Enfermería Global, v. 12, n. 1, 2013. Disponível em: <https://revistas.um.es/eglobal/article/view/165361>. Acesso em: 23 nov. 2018.

BEZERRA, F. N.; SILVA, T. M. da; RAMOS, V. P. Occupational stress of nurses in emergency care: an integrative review of the literature. Acta Paulista de Enfermagem, v. 25, n. SPE2, p. 151-156, 2012.

FERREIRA, R. G. Estresse do profissional de enfermagem no serviço noturno: uma questão de saúde. Revista Saúde e Desenvolvimento, v. 7, n. 4, p. 147-165-165, 25 jan. 2016.

KIRCHHOF, R. S. et al. Nível de estresse entre enfermeiros de um hospital da região Centro-Oeste - RS. Revista de Enfermagem da UFSM, v. 6, n. 1, p. 29-39, 30 mar. 2016.

LIMA, G. F.; BIANCHI, E. R. F. Estresse entre enfermeiros hospitalares e a relação com as variáveis sociodemográficas. Revista Mineira de Enfermagem, v. 14, n. 2, p. 210-218, 2010.

MAROCO, J.; GARCIA-MARQUES, T. Qual a fiabilidade do alfa de Cronbach? Questões antigas e soluções modernas? Laboratório de Psicologia, v. 4, n. 1, 17 nov. 2013. Disponível em: <http://publicacoes.ispa.pt/index.php/lp/article/view/763>. Acesso em: 6 jun. 2018.

OLIVEIRA, J. Da. de S. et al. Representacoes sociais de enfermeiros acerca do estresse laboral em um servico de urgencia. Revista da Escola de Enfermagem da USP, v. 47, n. 4, p. 984-989, 1 ago. 2013.

PETERSEN, R. S.; MARZIALE, M. H. P. Análise da capacidade no trabalho e estresse entre profissionais de enfermagem com distúrbios osteomusculares. Revista Gaúcha de Enfermagem, v. 38, n. 3, 16 nov. 2017. Disponível em: <https://seer.ufrgs.br/ RevistaGauchadeEnfermagem/article/view/67184>. Acesso em: 23 nov. 2018.

RIBEIRO, A. C.; SOUZA, J. F. de; SILVA, J. L. da. A precarização do trabalho no sus na perspectiva da enfermagem hospitalar. Cogitare Enfermagem, v. 19, n. 3, 30 set. 2014. Disponível em: <https://revistas.ufpr.br/cogitare/article/view/33034>. Acesso em: 6 jun. 2018.

TRETTENE, A. dos S. et al. Estresse em profissionais de enfermagem atuantes em Unidades de Pronto Atendimento. Boletim - Academia Paulista de Psicologia, v. 36, n. 91, p. 243-261, jul. 2016. 
WISNIEWSKI, D. et al. Satisfação profissional da equipe de enfermagem x condições e relações de trabalho: estudo relacional. Texto \&amp; Contexto - Enfermagem, v. 24, n. 3, p. $850-858$, set. 2015. 